\title{
Study of Factors Affecting Medical Incident: 3. Medicine Inspection
}

\author{
Yuka Miyachi*, Chika Nakayama, Takashi Horiba, Tomoaki Hino, Kazuyo Nagashiba, Miki Kato, \\ Masafumi Ohnishi, Hiroko Saito, Masato Isogai, Nami Sugiura, Keiko Matsuura, Taeyuki Oshima \\ College of Pharmacy, Kinjo Gakuin University, Nagoya, Japan \\ Email: *y-miyachi@kinjo-u.ac.jp
}

How to cite this paper: Miyachi, Y., Nakayama, C., Horiba, T., Hino, T., Nagashiba, K., Kato, M., Ohnishi, M., Saito, H., Isogai, M., Sugiura, N., Matsuura, K. and Oshima, T. (2020) Study of Factors Affecting Medical Incident: 3. Medicine Inspection. Pharmacology \& Pharmacy, 11, 9-16. https://doi.org/10.4236/pp.2020.111002

Received: December 3, 2019

Accepted: January 11, 2020

Published: January 14, 2020

Copyright $\odot 2020$ by author(s) and Scientific Research Publishing Inc. This work is licensed under the Creative Commons Attribution-NonCommercial International License (CC BY-NC 4.0). http://creativecommons.org/licenses/by-nc/4.0/

\begin{abstract}
It is the responsibility of pharmacists to ensure the safety of patients as pharmacist's professional duties expand into diverse directions. In recent years, the use of automated dispensing systems has increased; however, the inspection by pharmacists is the most important step in the prevention of medical errors. Comparing the inspection of hospital and community pharmacists may lead to an increased awareness of incident countermeasures. Therefore, eye tracking was performed for hospital and community pharmacists who conduct inspection, and the results revealed the factors that affect dispensing errors. One of the factors was the size of the dispensing space, especially if the space was small and the items needed were not easily accessible. In addition, differences in the inspection procedures among pharmacists were presumed to lead to possible dispensing errors due to oversights. Moreover, it was suggested that any subsequent work would flow more smoothly if a pharmacist checked the prescription and medication history management records at the beginning. Furthermore, it was thought that keeping a history of checks, such as recording any prescription checks in writing, would speed up finding the cause in the unlikely event that a dispensing error occurred. Accordingly, creating an environment in which one can concentrate without distractions during the inspections can lead to the prevention of dispensing error.
\end{abstract}

\section{Keywords}

Medicine Inspection, Dispensing Error, Eye Tracking, Pharmacist

\section{Introduction}

In recent years, hospitals and community pharmacy have become more aware of medical safety, and various medical accident prevention measures have been devised. According to a report by the Japan Council for Quality Health Care, ap- 
proximately $37.9 \%$ of errors involved medicines [1]. In 2016, the Ministry of Health, Labour and Welfare issued a notice regarding changes to barcode labeling for medicines. The new barcode incorporates the product code, expiry date, and serial number or code. Yamakita et al. reported that the implementation of the new barcode system contributed to decrease the number of error [2]. Moreover, according to a study by Sato et al. on how hospital pharmacists actually work, 1) Prescription checks based on patient information and the sharing of such information within a hospital contributed to accident prevention, 2) Preciseness in writing on envelope for medicines and labels can lead to accident prevention, 3) Timely provision of medicine information is useful for ensuring medical safety, and the involvement of hospital pharmacists leads to medical safety [3]. In recent years, various manufacturers have developed several dispensing devices as automated dispensing. New devices include a wide range of liquid medication dispensing machines, medicinal powder dispensing robots, packaged tablet checking support devices, and so on. However, it is financially and physically impossible to introduce such devices to all medical institutions. Further, even if one follows various medical accident prevention measures, it ultimately comes down to the pharmacist's eyes being indispensable.

Our laboratory has been using eye trackers in the picking and the inspection as well as the powder medication dispensing by hospital pharmacists to study factors affecting medical error [4] [5] [6] [7]. Eye tracking makes it possible to visualize and measure "what people are looking at", with eye tracking systems being widely used in the fields of ergonomics and psychology [8]. Eye tracking systems are also often used in medical settings, such as in studies on barrier-free of the elderly, and educational feedback comparing clinical nurses and nursing students [8] [9]. In this study, we considered the factors leading to medical incident using an eye tracking technology, capable of measuring a person's eye movement.

\section{Method}

\subsection{Subjects}

The subjects were 5 pharmacists working at Aichi Medical University Hospital $(\mathrm{HpP})$, and 5 pharmacists working at Suzuka Center Pharmacy or Suzuka Minami Pharmacy (PhP). The average number of years of working for $\mathrm{HpP}$ was $21.6 \pm 5.2$ years, and for $\mathrm{PhP}$ was $15.6 \pm 4.4$ years. The candidate chose at random from pharmacists who are working during more 5 years in hospital or pharmacy.

\subsection{Measurement Methods}

An eye tracking measurement experiment was conducted during inspection. Five prescriptions were checked at the hospital, and three prescriptions were checked at each pharmacy. Pharmacists in the same facility used the same prescriptions, envelope for medicines, and medicines during inspection. Gaze mea- 
surement device used was an eye mark recorder EMR-9 (NAC Image Technology Inc.). An example of the eye tracking video is shown in Figure 1.

\subsection{Gaze Points}

From the data collected, the gaze point during inspection was individually categorized. The gaze points used "Prescription (prescription check)" when the prescription looked during check the prescription, "Prescription (medicine check)" when the prescription looked during check the medicine, "Prescription (other)", "Medicine (name)", "Medicine (number)", "Medicine (other)", "Packaging (code/number)" the packaged medicine's the identification code/the packaged medicine's the number of tablets and capsules, "Packaging (number)" the total number of packages, and "Envelope for medicines".

\subsection{Pupil Diameter}

The left and right pupil diameters were averaged, and the mean pupil diameter for each the gaze category as divided was computed. The formula for obtaining the rate of change was as follows.

The rate of change $(\%)=$

(Mean pupil diameter for each category - Mean pupil diameter for all categories)

(Mean pupil diameter for all categories)

\subsection{Statistical Analysis}

Differences in each parameter were statistically evaluated by the Mann-Whitney $\mathrm{U}$ test. All differences were considered significant when $p<0.05$.

\subsection{Ethics Statements}

This study is approved by the Kinjo Gakuin University Ethical Review Board (No. H17015, January 31 2018).

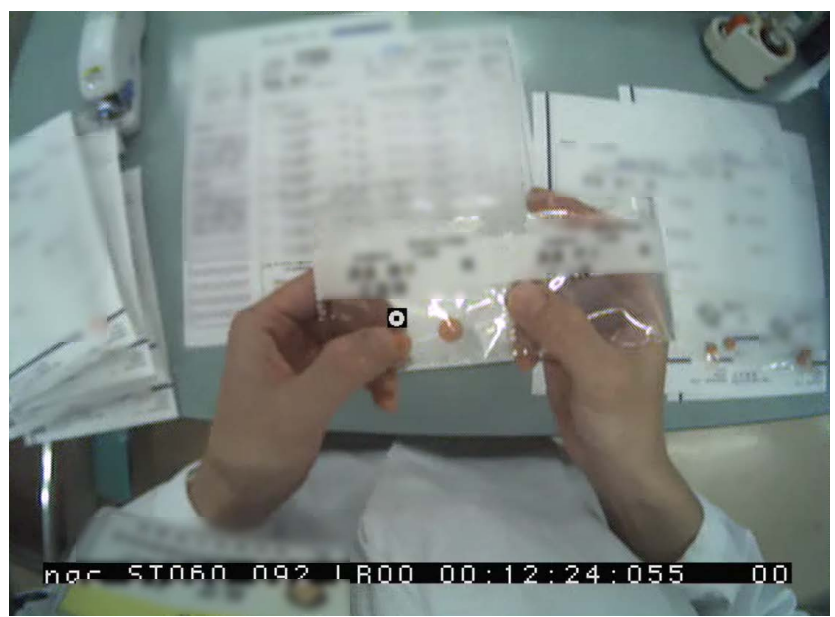

Figure 1. The eye tracking video (the white circles represent the gaze point). 


\section{Result}

\subsection{Eye Tracking Measurement Experiment}

Table 1 shows the prescriptions checked by $\mathrm{HpP}$ and $\mathrm{PhP}$. There were no significant differences in these prescriptions except for the dosage days.

Figure 2 shows the gaze rate during inspection per prescription. The gaze rate of "Prescription (medicine check)" of $\mathrm{HpP}(19.7 \% \pm 7.2 \%)$ was significantly higher than $\mathrm{PhP}(10.5 \% \pm 7.2 \%)(p<0.05)$. The gaze rate of "Packaging (number)" of $\mathrm{PhP}(14.3 \% \pm 5.9 \%)$ was significantly higher than $\mathrm{HpP}(8.1 \% \pm 4.2 \%)(p$ $<0.05)$. The gaze rate of "Prescription (medicine check)" of $\mathrm{HpP}(9.8 \% \pm 8.9 \%)$ was tended to be higher than $\mathrm{PhP}(6.2 \% \pm 3.4 \%)$. The gaze rate of "Packaging (code/number)" of HpP $(29.9 \% \pm 10.1 \%)$ and $\mathrm{PhP}(35.5 \% \pm 12.4 \%)$ was the highest gaze rate of all the items.

\subsection{Changes in Pupil Diameter}

Figure 3 shows the rate of change in pupil diameter for $\mathrm{HpP}$ and $\mathrm{PhP}$. The rate of change of pupil diameter of "Prescription (prescription check)" was $2.1 \% \pm$ $5.0 \%$ and $-4.3 \% \pm 4.0 \%$ for $\mathrm{HpP}$ and $\mathrm{PhP}$, respectively. The pupil diameter of "Prescription (medicine check)", Prescription (other)", "Medicine (name)", "Medicine (number)", "Medicine (other)", and "Envelope for medicines" for $\mathrm{HpP}$ and $\mathrm{PhP}$ were tended to mydriasis. The rate of change of pupil diameter of "Packaging (code/number)", was $-9.5 \% \pm 4.6 \%$ and $-5.7 \% \pm 4.1 \%$ for $\mathrm{HpP}$ and $\mathrm{PhP}$, both of which the miosis. The pupil diameter of "Packaging (number)" of $\mathrm{PhP}(3.3 \% \pm 2.7 \%)$ was a significantly greater than $\operatorname{HpP}(-2.6 \% \pm 2.2 \%)(p<$ $0.05)$.

\section{Discussion}

In recent years, pharmacists' professional duties have expanded, and dispensing errors have become various types. For example, patients can become mentally stressed when they are given the wrong medicine, even before taking it. Since this type of stress may reduce adherence, it is important to consider multifaceted improvement measures. In this study, we conducted eye tracking measurements of inspections by $\mathrm{HpP}$ and $\mathrm{PhP}$ to find factors that affect dispensing errors, and consider human error countermeasures.

Table 1. The prescriptions.

\begin{tabular}{lccc}
\hline & $\mathrm{HpP}(\mathrm{n}=5)$ & $\mathrm{PhP}(\mathrm{n}=5)$ \\
\hline & Working experience (year) & $21.6 \pm 5.2$ & $15.6 \pm 4.4$ \\
& Number of picking medicines & $2.0 \pm 1.3$ & $3.7 \pm 0.9$ \\
Per prescription & $\begin{array}{l}\text { Numberof medicines } \\
\text { in one dose package } \\
\text { Number of total medicines }\end{array}$ & $6.4 \pm 2.1$ & $8.2 \pm 2.2$ \\
& Dosage days & $7.4 \pm 2.7$ & $11.8 \pm 2.7$ \\
& & $32.7 \pm 6.6^{*}$ \\
\hline
\end{tabular}

Mesn $\pm \mathrm{SD}(\mathrm{n}=5) ;{ }^{*} p<0.05$. 


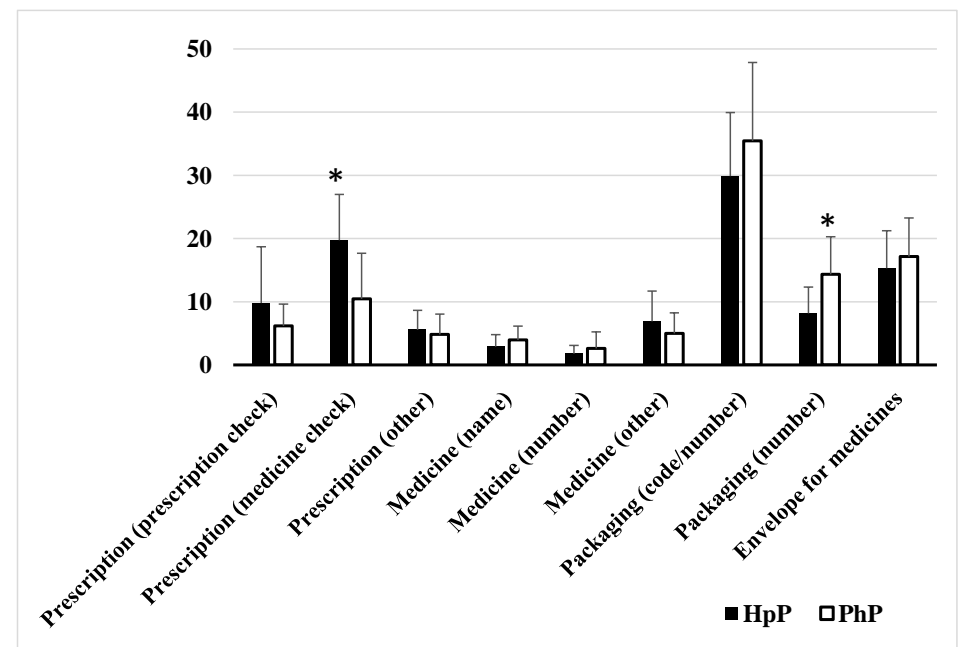

Figure 2. The gaze rate per prescription (\%). Mean \pm SD $(\mathrm{n}=15-25) ;{ }^{\star} p$ $<0.05$.

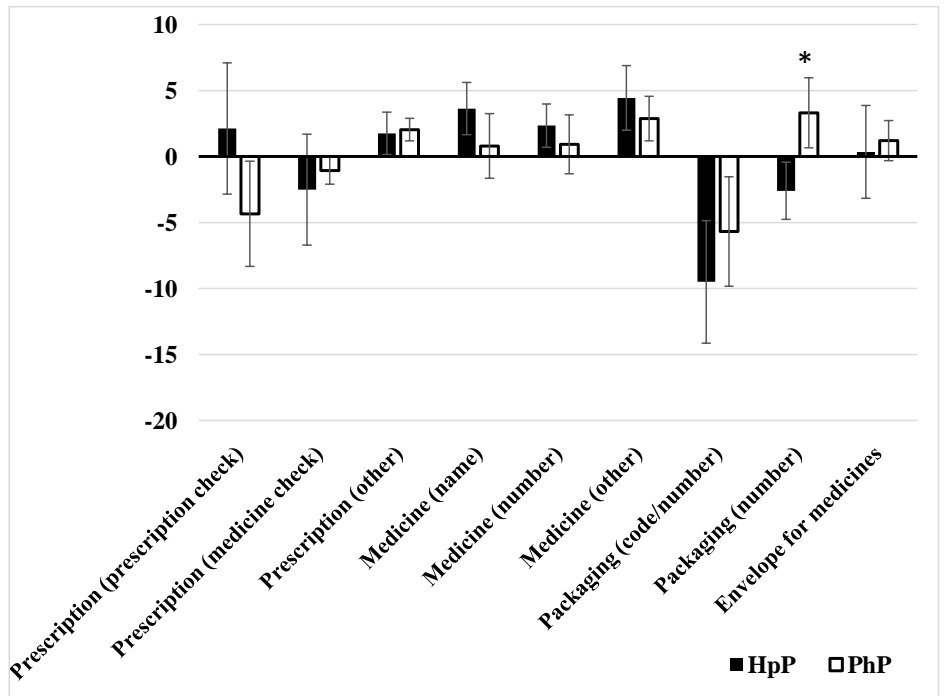

Figure 3. The rate of change in pupil diameter (\%). Mean \pm SD $(n=5)$; ${ }^{*} p<0.05$.

The incidence of errors at the institutions in which we conducted the research in 2018 was approximately $1.6 \%$ at the $\mathrm{HpP}$ and approximately $0.94 \%$ at the $\mathrm{PhP}$. However, accidents were approximately $0.004 \%$ at the HpP and approximately $0.03 \%$ at the PhP. Therefore, it was shown that, errors were discovered by the inspection of the $\mathrm{PhP}$ which was less than the $\mathrm{HpP}$.

In the results of the eye tracking, we indicated that $\mathrm{PhP}$ tended to have lower gaze rate compared to $\mathrm{HpP}$ in "Prescription (prescription check)", and there was a significant difference in "Prescription (medicine check)". Further, the rate of change in pupil diameter of "Prescription (prescription check)" resulted in a significant toward contraction for $\mathrm{PhP}$. In addition to prescription checks, pharmacy pharmacist's work involves checking the medication history management records, medical fees, receipts, and so on [10]. These results are inferred to 
be the attention points which were dispersed; it was deduced that the gaze rate was low and the pupil diameter was also reduced. Moreover, it was considered that the resulting number of accidents mentioned above was also affected. It was necessary to prepare a working environment so that I could concentrate on the inspection. For example, confirmer of the medical fees can do by the medical clerk; thus, it is thought that inspection's pharmacist could concentrate on "confirm prescription".

The gaze rate of "Packaging (number)" was significantly higher in PhP. Further, the rate of change of pupil diameter on "Packaging (number)" was significantly higher for $\mathrm{PhP}$ than $\mathrm{HpP}$. It was affected that the prescriptions inspected by $\mathrm{PhP}$ had significantly longer dosage days in this study.

It was considered that the pupil diameter in "Packaging (code/number)" exhibited a changed toward contraction for both $\mathrm{HpP}$ and $\mathrm{PhP}$, because contraction occurs due to short-sighted work when checking the code and number [11].

Moreover, at the institutions in which we conducted the research, the inner bags and rubber bands required for inspection were not easily accessible. As a result, the pharmacists had to move every time they need those extra work. It has been reported that interruptions during work cause incidents [12] [13] [14]. It was thought that looking away from the prescription or medicine during inspection is presumed to result in loss of concentration and reduced attention. There were also cases in which the space for inspecting was small, and the prescription and envelope for medicines were inspected on top of a computer keyboard. Inspecting on uneven surfaces may lead to serious mistakes such as dropping or loss of medicines, picking up the wrong prescriptions, and so on. One of the measures for preventing dispensing errors and accidents caused by human error is organizing the dispensing room [15]. Therefore, it was suggested that it's necessary to maintain a well-organized inspecting space and dispensing room.

Moreover, there were differences in the working procedures; for example there were pharmacists who did not check prescriptions first and others did not check the medication history records during prescription checks, and so on. It was thought that the subsequent work will flow more smoothly if items such as prescription and medication history management record checks are performed first. Moreover, it has been reported that standardizing the series of work procedures could prevent mistakes and oversights [16]. Consequently, reconsideration of the work procedures during inspection should be considered as an incident countermeasure.

Furthermore, some pharmacists record the checked content by writing it down to prescriptions. In the unlikely event that a dispensing error occurs, it is important to discover medicine before the medicine is actually taken [17] [18]; thus, having a record at the inspection may be an important key in any subsequent investigation of the cause.

According to the Japan Council for Quality Health, the most common cause of near-miss incidents in pharmacies is reported to be "failure to check", as well as human factors such as "busy working conditions"; thus, the causes of medical 
error may be complicated [19]. Our study using eye trackers, can help to elucidate each institution's idiosyncrasies, and lead to clarification of the causes of incidents as well as prevention of medical errors in the future.

\section{Acknowledgements}

We would like to thank the pharmacy staff of the Department of Pharmacy, Aichi Medical University Hospital, Suzuka Minami Pharmacy and Suzuka Center Pharmacy who cooperated in this study.

\section{Conflicts of Interest}

The authors declare no conflicts of interest regarding the publication of this paper.

\section{References}

[1] Pharmaceuticals and Medical Devices Agency (2016) PMDA Alert for Proper Use of Drugs. No. 337.

[2] Yamakita, K., Kanno, N., Ohmichi, H., Kondo, Y. and Umesato, Y. (2013) Use and Problems of a New Bar Code (GS1 DataBar) in Health Insurance Pharmacies: From the Standpoint of the Traceability of Pharmaceuticals and Prevention of Their Drug-Dispensing Errors. Journal of the Japan Society for Healthcare Administration, 50, 189-197.

[3] Sato, H. and Endo, K. (2007) The Role and Responsibility of Hospital Pharmacist to Prevent Mix-Up of Pharmacist Practices. IRYO, 61, 673-675.

[4] Oshima, T., Kato, A., Saito, S., Uchida, M., Maeda, S., Onishi, M., Takakuwa, Y. and Hasegawa, T. (2011) Study on Factor Analysis on Medical Error (1) Influence of Experience of the Pharmacist to Dispensing. Journal of Ergonomics in Occupational Safety and Health, 12, 1-7.

[5] Oshima, T., Kato, A., Saito, S., Uchida, M., Maeda, S., Onishi, M., Takakuwa, Y. and Hasegawa, T. (2011) Study on Risk Management of Drugs in the Hospital Pharmacy (2) Influence of Experience of the Pharmacist to Final Inspection. Journal of Ergonomics in Occupational Safety and Health, 12, 8-14.

[6] Miyachi, Y., Nakayama, C., Nagashiba, K., Kinoshita, K., Takeuchi, M., Ohnishi, M., Saito, H. and Oshima, T. (2018) Study of Factors Affecting Medical Incident: 1. Dispensing. Pharmacology \& Pharmacy, 9, 527-535. https://doi.org/10.4236/pp.2018.912041

[7] Miyachi, Y., Nakayama, C., Funahashi, S., Shimada, H., Takeuchi, T., Ohnishi, M., Saito, H. and Oshima, T. (2019) Study of Factors Affecting Medical Incident: 2 Powdered Medication Dispensing. Pharmacology \& Pharmacy, 10, 309-317. https://doi.org/10.4236/pp.2019.106025

[8] NAC Image Technology Inc. (2019) https://www.nacinc.jp/

[9] Yoneda, T., Itami, K., Kawabata, Y., Seki, K., Kubota, Y., Kito, Y., Matunami, M., Yasui, A., Matuda, A., Umemoto, N., Shimizu, F., Kuroda, Y. and Maesako T. (2017) The Risk Awareness Clinical Nurses on Watching an Elderly-Patient Walking along a Ward Hallway: Comparison between Nursing Student and Nurse. Journal of Human Nursing Studies, 15, 1-10.

[10] Sakakibara, M. (2015) Activity of Pharmacy Pharmacist in Community Based Intergrated Care System. Journal of Japanese Society for Parenteral and Enteral Nutri- 
tion, 3, 789-792.

[11] Hara, N. (2012) Pupillographic Stress, Arousal and Emotion Assessments in Visual Stress. Japanese Journal of Visual Science, 33, 47-51.

[12] Takeuchi, H., Kanazaki Y., Yamamoto, H., Makino, M., Saito, D. and Konaga, E. (2003) Prevention of Medication Errors by Analyzing Incident Reports. The Japanese Journal of the National Medical Services, 57, 558-561.

[13] Kasai, Y., Ishikawa, Y., Shuda, A. and Shijiki, Y. (2016) Factors Associated with Medical Near-Miss, Incidents and Accidents by Nurses Performing Shift Work. The Journal of Japan Academy of Health Sciences, 19, 14-23.

[14] Yokoi, Y. (2002) The Circumstances of Nursing Works and Medical Accidents. Reliability Engineering Association of Japan, 24, 127-133.

[15] Tachi, T., Teramachi, H., Tamura, K., Komada, N., Shiga, H., Imai, K. and Tsuchiya, T. (2012) Analysis of Relationship between Environmental Improvements of Pharmacy and Human Error in Preventive Measures for Dispensing Mistakes. Journal of Pharmaceutical Health Care and Sciences, 38, 513-521. https://doi.org/10.5649/jjphcs.38.513

[16] Nakamichi, N., Kiura, M. and Yamada, T. (2012) Behavior Analysis of Prescription Checking Based on Eye Information. The Institute of Electronics, Information and Communication Engineers Technical Report, 85, 1-4.

[17] Yoshida, K., Hayashi, K., Kanda, A., Doi, Y., Otani, K. and Iseki, K. (2012) Evaluation of Risk Priority of the Dispensing Process by Using a Risk Management System in Community Pharmacies. Japanese Journal of Drug Informatics, 14, 21-25.

[18] Taga, S., Kimura, M. and Yoshimura, T. (2018) Effect of Prevention of Dispensing Errors by Utilizing Picking Support System in Inspection. Journal of Japanese Society of Hospital Pharmacists, 54, 416-420.

[19] Japan Council for Quality Health Care Division of Adverse Event Prevention (2018) Project to Collect and Analyze Pharmaceutical Near-Miss Event Information 2017 Annual Report. 